\title{
Clinical Development of Src Family Kinase Inhibitors in Malignant Mela- noma
}

\author{
Iain R.J. Macpherson*,1, Rob J. Jones ${ }^{2}$ and T.R. Jeffry Evans ${ }^{2}$ \\ ${ }^{I}$ Department of Medical Oncology, The Beatson West of Scotland Cancer Centre, 1053 Great Western Road, Glasgow \\ G12 OYN, United Kingdom \\ ${ }^{2}$ Centre for Oncology and Applied Pharmacology, University of Glasgow, Cancer Research UK Beatson Laboratories, \\ Garscube Estate, Switchback Road, Glasgow G61 1BD, United Kingdom
}

\begin{abstract}
Currently available systemic therapies for malignant melanoma are unsatisfactory and there is an urgent need for effective and well tolerated drugs for use in both early and advanced disease. The Src family of cytoplasmic tyrosine kinases (SFKs) have been implicated in the regulation of many of the hallmarks of malignancy making them attractive targets in solid tumours including melanoma. The first generation of selective SFK inhibitors to enter the clinic (AZD0530, dasatinib, bosutinib) have demonstrated safety, tolerability and target modulation in phase I trials. Phase II trials in patients with advanced melanoma are now planned in the USA and Europe. Here we discuss the rationale for, and challenges facing, the successful development of SFK inhibitors in melanoma. Furthermore, as dasatinib is also a potent inhibitor of the receptor tyrosine kinase (RTK), c-Kit, we reconsider the utility of targeting this kinase in the light of recent molecular epidemiological data.
\end{abstract}

\section{INTRODUCTION}

Malignant melanoma remains the deadliest of skin cancers. Its incidence has steadily risen with approximately 60 000 new cases diagnosed in Europe in 2000 [1]. Melanoma detected whilst localised to its site of origin is amenable to cure by complete surgical excision. In such cases the risk of occult micrometastatic disease and subsequent relapse is best predicted by pathological factors such as the Breslow thickness (depth of invasion) and the presence of epidermal ulceration. Disease involving regional lymph nodes confers a significantly worse prognosis although cure remains possible with radical lymph node dissection. The presence of distant metastases confers a bleak prognosis with a median survival of six to nine months [2]. Current systemic therapies for melanoma are poorly tolerated and / or of limited efficacy, both in the peri-operative and advanced disease setting [3, 4]. Effective and well tolerated therapies for use in both early and advanced disease are therefore urgently required.

\section{TYROSINE PHOSPHORYLATION IS INCREASED IN MELANOMA}

The modification of proteins by the addition of phosphate groups is a major activating event in signal transduction and is involved in the regulation of nearly every aspect of cell biology. Abnormally high phosphorylation of proteins on tyrosine residues is a common finding in malignant cells and results from the increased activity of receptor or nonreceptor tyrosine kinases (RTKs) or from the decreased activity of tyrosine phosphatases [5].

Immunohistochemical analysis of melanoma surgical specimens with a pan-phosphotyrosine antibody revealed a

*Address correspondence to this author at the Department of Medical Oncology, The Beatson West of Scotland Cancer Centre, 1053 Great Western Road, Glasgow G12 0YN, United Kingdom;

E-mail: iain.macpherson@NorthGlasgow.Scot.NHS.UK significant increase in phosphotyrosine staining in melanomas in comparison to adjacent normal melanocytes or melanocytic nevi [6]. Strikingly, the frequency and intensity of phosphotyrosine staining increased in a stepwise fashion with the greatest phosphorylation occurring in later (vertical growth phase and metastatic melanoma) rather than earlier (nevi, radial growth phase melanoma) lesions. This elevation in phosphotyrosine content may be explained by increased activity of receptor (e.g. c-Met, EGFR, FGFR, Ephrins) and non-receptor (e.g. SFKs, FAK) tyrosine kinases and by the reduced activity of tyrosine phosphatases [6-8]. However, the relative contribution of individual kinases or phosphatases in melanoma is poorly understood. Here we focus specifically on the potential role of SFKs but also consider, where relevant, c-Kit and other tyrosine kinases susceptible to inhibition by dasatinib, AZD0530 and / or bosutinib.

\section{SRC-FAMILY KINASES (SFKS) IN CANCER}

Spanning the $20^{\text {th }}$ century, the venerable position of the viral oncogene $v$-Src and its cellular homologue $c$-Src (hereafter referred to as $S r c$ ) in the developing fields of cell and cancer biology has been reviewed elsewhere [9]. Src (Fig. 1) is the prototypic member of a family of nine cytoplasmic tyrosine kinases and displays significant functional redundancy with the two other ubiquitously expressed SFKs, Yes and Fyn [10]. Expression of the remaining SFKs (Lyn, Lck, Hck, Blk, Fgr and Yrk) is restricted primarily to haematopoietic cells. SFKs are basic components of the cell signalling machinery and are involved in diverse signalling pathways regulating growth, survival, motility and adhesion which originate from receptor tyrosine kinases, integrins, cadherins, cytokine receptors and G-protein coupled receptors [10].

The kinase activity of Src is regulated by several mechanisms which have in common the ability to influence the stability of inhibitory intra-molecular interactions [11]. When phosphorylated, Src tyrosine-530 forms an intra- 
molecular interaction with the $\mathrm{SH} 2$ domain whilst a further intra-molecular interaction occurs between the SH3 domain and a short stretch of amino acids between the $\mathrm{SH} 2$ domain and kinase domain. In this conformation not only is the Src kinase domain inaccessible but access of potential binding partners to the protein-protein interacting $\mathrm{SH} 2$ and $\mathrm{SH} 3$ domains is sterically hindered. v-Src, the oncoprotein isolated from Rous Sarcoma Virus, lacks the C-terminal regulatory sequences present in Src and so is constitutively active [12].

Src expression and / or activity is upregulated in a variety of human tumours [13]. The largest body of evidence exists for colorectal cancer in which the activity of Src is elevated in tumour specimens when compared to adjacent normal mucosa and the degree of activity increases in parallel with advancing stage of disease [14-16]. However, elevated Src kinase activity has also been reported in pathological specimens from human breast [17, 18], gastric [19], pancreatic [20] and oesophageal cancers [21].

\section{SFK EXPRESSION AND ACTIVITY IS INCREASED IN MELANOMA}

Importantly, Daud and colleagues have determined the in-situ activity of SFKs in melanoma by immunohistochemical analysis of tumour biopsies with an anti-Src phosphotyrosine-419 antibody [23]. It should be noted that, due to homology within the activation loop, phosphospecific antibodies raised against Src phospho-tyrosine-419 do not discriminate between Src and other SFKs. Utilising this antibody, SFK activity was detected in $48 \%$ of melanomas with the strongest staining occurring in metastatic lesions.

Complementary in vitro data suggest that Yes rather than Src may be the most significant contributor to the elevated SFK activity observed in melanoma. Specifically, Loganzo reported 5-10 fold elevation of Yes activity, but not Src activity, in 18 of 20 melanoma cell lines in comparison to normal melanocytes [24]. Similarly, Gallick and colleagues have described elevated Yes but not Src activity in metastatic versus poorly metastatic melanoma cell lines [25]. In addition, O'Conner reported no increase in Src activity in 6 melanoma cell lines in comparison to normal melanocytes [26]. However, the activity of Src itself also varies considerably among melanoma cell lines $[27,28]$ and among clinical specimens, including variability of Src activity among metastatic deposits within a single patient [29]. Finally, the elevated SFK activity seen in the Xiphophorus model of melanoma appears to be attributable to Fyn [30]. Taken together these data suggest that activation of SFKs may be a common occurrence in melanoma, although the precise contribution of individual family members may vary and remains ill-defined.

\section{MECHANISMS OF SFK ACTIVATION IN MELA- NOMA}

Data obtained in melanoma cell lines, viewed in concert with data obtained in other tumour types, support the existence of several non-mutually exclusive events leading to SFK activation.

\section{Increased SFK Protein Levels}

A distinction may be made between increased SFK kinase activity which is directly proportional to an increase in protein levels versus an increase in specific activity i.e. an increased kinase activity which occurs in the absence of, or disproportionately to, a change in protein levels and which reflects post-translational regulation. For example, the increased Yes activity observed in 18 of 20 melanoma cell lines was attributed to a parallel increase in protein levels [24]. This was associated with an increase in mRNA transcripts without evidence of genomic amplification. However, other workers have described alterations in specific SFK activity occurring independently of alterations in protein levels, supporting the existence of additional levels of regulation in melanoma $[25,31]$.

\section{Mutational Activation}

A C-terminal truncating and activating $\operatorname{Src}$ mutation has been reported in a subset of colorectal cancers [32]. However, this finding has not been widely replicated [33-35] suggesting that mutational activation of Src is an infrequent event in colorectal cancer. This mutation has either not been detected in other solid tumours, including transitional cell carcinoma [36] or ovarian carcinoma [37], or has been detected at very low frequency, as in endometrial cancer [37]. Loganzo et al detected no mutations in the C-terminal regulatory region or, indeed, any coding region, of Yes in 20 melanoma cell lines tested [24]. Activating mutations, at least for Yes, would therefore appear unlikely to be a common mechanism of SFK activation in melanoma.

\section{Downstream of Activated RTK Signalling}

Typically, on encountering its cognate ligand, a RTK undergoes dimerisation and transphosphorylation of tyrosine residues within the cytoplasmic domain. These residues form high-affinity binding sites which the SFK SH2 domain favours in preference to the lower affinity intra-molecular tyrosine-530 interaction hence resulting in simultaneous re-

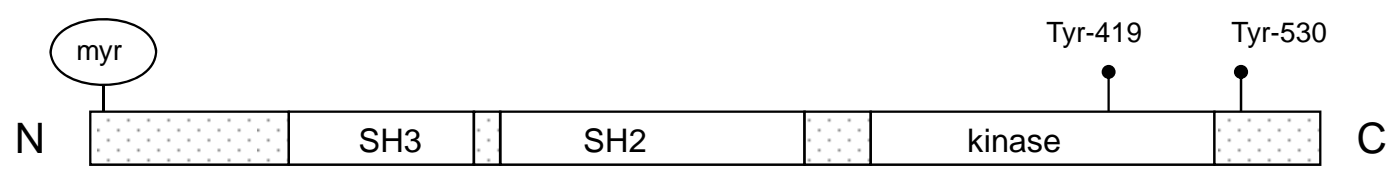

Fig. (1). Structure of Src: Src, the prototypic SFK, has a modular structure comprising an N-terminal unique region containing a myristylation site (myr) which is essential for membrane localisation, an SH3 domain, an SH2 domain, a kinase domain and a C-terminal regulatory sequence [11]. The SH2 and SH3 domains mediate protein-protein interaction; the SH3 domain binds proline rich sequences whilst the SH2 domain binds regions containing phosphorylated tyrosines. Within the activation loop of the kinase domain tyrosine-419 undergoes autophosphorylation and is therefore often used as a readout of Src kinase activity. Tyrosine-530, lies in the C-terminal regulatory region and, in contrast to tyrosine-419, its phosphorylation is associated with inactivation of Src [22]. 


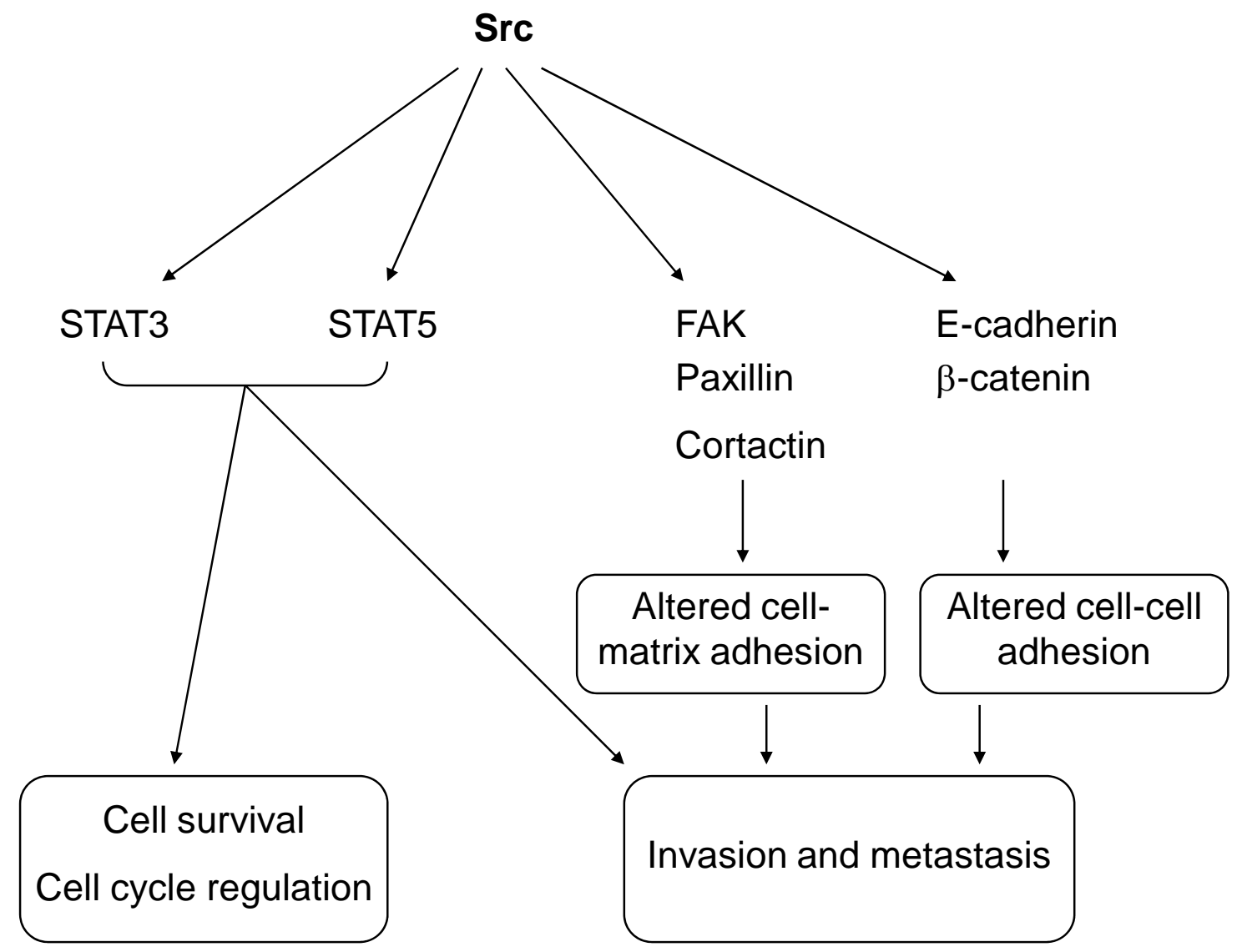

Fig. (2). Schematic diagram of key SFK-dependent pathways in melanoma.

cruitment and activation of the SFK [38]. Therefore, elevated SFK activity may be secondary to activation of an upstream RTK to which it is coupled $[39,40]$.

To date several RTKs have been implicated in the development or progression of melanoma, including c-Kit (discussed below), c-Met [41], EGFR [42, 43], and FGFR [7]. A causal role for RTK signalling in melanomagenesis is supported by the frequent development of melanomas in transgenic mice overexpressing hepatocyte growth factor (the ligand for c-Met) [44]. Furthermore, the Xiphophorus melanoma receptor kinase (Xmrk) is responsible for a hereditary melanoma syndrome in the fish Xiphophorus (recently reviewed in [45]). Xmrk is a mutated form of the fish orthologue of EGFR which has acquired 2 mutations in the extracellular domain leading to constitutive dimerisation and ligand-independent activation [46]. In both of these models the melanomas are highly invasive, reminiscent of the observation that phosphotyrosine content is generally highest in vertical growth phase and metastatic lesions in human melanoma.

Several observations directly support RTK signalling as one mechanism of SFK activation in melanoma. First, the constitutive activation of a SFK in 586 mel melanoma cells was abrogated by expression of a dominant negative FGF receptor [31]. Protein levels were unchanged indicating that the reduction was in specific activity. Second, Yes (but not Src) has been implicated downstream of NGF signalling in melanoma cell lines and its activity correlated with their invasive and metastatic potential [25]. Third, Xmrk recruits and activates Fyn (although not Yes or Src) in fish [30] or mammalian melanocytes [47]. Finally, Src is activated and forms a complex with EGFR upon stimulation of A375 human melanoma cells with EGF [48].

\section{Displacement of Inhibitory Intramolecular Interac- tions}

Analogous to the action of activated RTKs, cytoplasmic proteins may similarly displace inhibitory SFK intramolecular interactions if they contain sequences which will bind the SFK SH2 or SH3 domains with greater affinity. For example, focal adhesion kinase (FAK), a cytoplasmic TK implicated in the regulation of cell adhesion, migration and survival, is localised to integrin-rich cell-matrix contacts and becomes phosphorylated on integrin ligation to create a high affinity $\mathrm{SH} 2$ domain binding motif thus recruiting and activating Src [49]. A consensus SH3 domain binding motif also contributes to FAK's ability to activate Src [50]. As described below, FAK expression and / or phosphorylation is often upregulated in melanoma cell lines suggesting that this may be an important mechanism of SFK activation [51, 52].

\section{Reduced Phosphorylation of Regulatory Tyrosine-530}

The phosphorylation state of Src tyrosine-530 acts as a molecular switch with phosphorylation favouring inactivity and de-phosphorylation favouring activation. Reduced phosphorylation of tyrosine-530 may occur via a reduction in the expression of C-terminal Src Kinase (Csk) as has been reported in some hepatocellular cancers [53] or by the increased expression of phosphatases. For example, PTP-1B dephosphorylates tyrosine-530 and activates Src in several 
breast cancer cell lines [54]. Interestingly, this phosphatase is also overexpressed in several melanoma cell lines [8] whilst the increased specific activity of Src in melanocytes in comparison to fibroblasts was associated with a reduction in phosphorylation of tyrosine-530 [26].

Other mechanisms of Src activation in cancer have also been proposed including elevated $\mathrm{N}$-myristoyltransferase activity, altered intracellular targeting, altered proteasomal degradation and altered serine/threonine phosphorylation $[55,56]$. However, no direct data exist to link these mechanisms to melanoma.

\section{CONSEQUENCES OF SFK SIGNALLING IN MELA- NOMA}

A growing body of evidence indicates that SFKs can regulate many processes relevant to neoplasia including cell cycle progression, survival, motility and invasion (Fig. 2) $[55,56]$.

\section{Invasion and Metastasis}

One of the major contributions of SFK signalling in cancer may be to confer an invasive or metastatic phenotype. Indeed, many SFK substrates including FAK, cortactin, $\mathrm{p} 130^{\mathrm{cas}}$ and $\mathrm{p} 120$-catenin are proteins directly implicated in the regulation of cell adhesion and migration [57]. In particular, a significant body of evidence has been generated to support a cooperative and critical role for Src and FAK in cancer cell migration [57]. Consequently, it is not surprising that several lines of evidence support a central role for the Src-FAK axis in the migration and invasion of melanoma cells. For example, SFK activity correlated with the metastatic capacity of melanoma cell lines $[25,58]$. Similarly, both the level of FAK expression and the degree of phosphorylation of FAK at tyrosine-379 and -576 correlated with invasiveness and / or motility of melanoma cell lines [51, 52]. Consistent with these observations was the demonstration that the ability of exogenous Xmrk to render poorly motile murine melanocytes highly motile required the coordinated action of Fyn and FAK [59]. Furthermore, the migration and invasion of C8161 melanoma cells was abrogated by expression of a dominant negative FAK protein [52]. The requirement for SFK-FAK signalling in melanoma is further highlighted by the finding that treatment of melanoma cell lines with dasatinib blocked phosphorylation of FAK and completely inhibited the motility and invasiveness of A2058 melanoma cells [60].

\section{Increased Proliferation}

Studies in both fibroblasts and epithelial cells have identified a role for SFKs in cell cycle progression, particularly in the context of growth factor signalling [10]. In keeping with these observations the proliferation of both normal melanocytes and melanoma cells in response to FGFR signalling is associated with SFK activity [31]. Furthermore, the transformation and proliferation of mouse melanocytes in response to expression of Xmrk is mediated by Fyn [47]. Despite this, in most tumour types studied only a subset of cell lines appear to depend on SFK activity for proliferation. For example, concentrations of dasatinib which fully blocked Src activity inhibited proliferation in only 2 of 12 colon cancer cell lines tested [61]. Similarly, pharmacologically relevant concentrations of dasatinib did not inhibit proliferation in 13 of 24 breast cancer cell lines and 4 of 15 lung cancer cell lines [62]. In-keeping with these observations, antiproliferative effects of AD0530 were also observed in only a minority of cell lines treated [63]. The extent to which SFK inhibition might inhibit the proliferation of melanoma cells is therefore uncertain. Existing data obtained in melanoma cell lines suggest that, in common with other solid tumours, the anti-proliferative effects of SFK inhibitors may be quite variable [23, 60, 64]. Smalley and Herlyn have recently suggested that vertical growth phase and metastatic melanoma cells may be relatively insensitive to the anti-proliferative effects of SFK inhibition in comparison to radial growth phase cells. This intriguing but preliminary observation would clearly have implications for development of SFK inhibitors and requires further investigation [64].

\section{Enhanced Survival}

Accumulating evidence supports an important prosurvival role for Src-dependent activation of the signal transducer and activator of transcription (STAT) transcription factors in melanoma. First, expression of a dominant negative Stat 3 protein caused apoptosis of B16 melanoma cells in vitro and in vivo [28]. Stat3 was subsequently shown to be constitutively activated in 16 of 18 melanoma cell lines [28] and in the majority of tumour specimens examined [23, 28]. Second, in cell lines and tissue specimens there was a correlation between Src activity and phosphorylation / activation of Stat3 whilst pharmacological inhibition of SFK activity caused a reduction in Stat3 activity in cell lines [23, 28]. Third, either dominant negative Stat 3 expression or pharmacological SFK inhibition induced apoptosis in JW and A2058 melanoma cell lines and this was associated with inhibition of expression of the anti-apoptotic genes $\mathrm{Bcl}-X_{L}$ and $\mathrm{Mcl}-1$ [28]. Finally, Stat5 was hyperphosphorylated in $62 \%$ of melanoma metastases tested [48]. Stat5, like Stat3 was also activated by Src and inhibition of Stat5 expression caused enhanced apoptosis in A375 and BLM melanoma cells which, in this case, was associated with reduced expression of Bcl-2 [48].

\section{Enhancement of Chemosensitivity}

SFK inhibitors have been reported to enhance cell kill or to overcome drug resistance in a variety of models [65-67]. In this context, dasatinib has been reported to enhance the sensitivity of melanoma cell lines to platinum and topoisomerase I inhibitors [23].

\section{SFK Signalling in Stromal Cells}

Regardless of the consequences of inhibiting SFK signalling in melanoma cells, SFK inhibitors may independently exert effects by interfering with SFK-dependent functions in stromal cells. For example, angiogenesis, a promising target for melanoma therapeutics, may be susceptible to SFK inhibition [68-71]. In addition, lytic bone metastases which are a common feature of advanced melanoma might be ameliorated by inhibiting SFK signalling in osteoclasts [72, 73].

\section{SFK INHIBITORS IN CLINICAL DEVELOPMENT}

\section{Dasatinib}

Dasatinib (BMS-354825; Bristol-Myers Squibb, Princeton, $\mathrm{NJ}$ ) is a potent, orally active, ATP-competitive inhibitor of 5 kinases / kinase families: SFKs, c-Kit, Abl, PDGFR and 
EphA2 [74]. Dasatinib inhibits both Abl and c-Kit more potently than imatinib although whether this might translate into greater clinical efficacy in CML and GIST is the subject of ongoing clinical investigation [75]. Crucially, however, dasatinib retains activity against 21 of 22 tested imatinibresistant Bcr-Abl mutants [76-78] as well as imatinibresistant c-Kit mutants [79, 80]. Molecular insights provided by the crystal structure of the dasatinib-Abl complex demonstrate less stringent conformational requirements in the activation loop for dasatinib-binding in comparison to imatinib. In addition, dasatinib does not form critical interactions with most of the P-loop residues whose mutation interferes with imatinib binding [81]. Similarly, less stringent conformational requirements may also explain the activity of dasatinib against imatinib-resistant forms of c-Kit [80].

Preclinical studies demonstrated efficacy of dasatinib against Bcr-Abl positive leukemias [76] and, on the basis of efficacy in phase I and II trials [82-86], dasatinib was granted approval by the FDA in 2006 for use in patients with CML or Philadelphia chromosome positive acute lymphoblastic leukaemia with resistance to or intolerance of imatinib. The development and use of dasatinib in haematological malignancy has been extensively reviewed elsewhere [87].

The development of dasatinib in solid tumours is less advanced. However, data obtained in preclinical models suggest dasatinib may interfere with several of the hallmarks of malignancy. Specifically, pharmacologically achievable concentrations of dasatinib have influenced proliferation [61, 62, 88,89 ], survival [88-90], migration and invasion [61, 88-91], metastasis [92], and drug sensitivity [23] in a variety of solid tumour preclinical models. In melanoma Jove and colleagues have presented data showing treatment of cell lines with dasatinib inhibited phosphorylation of FAK, caused rounding and altered cell matrix adhesion and could completely abolish invasion and migration [60]. Dasatinib has also been reported to inhibit proliferation of melanoma cell lines and to synergise with platinum and topoisomerase I inhibitors [23].

Preliminary results of a phase I study of dasatinib in patients with GIST and other solid tumours including malignant melanoma have been presented [93]. In contrast to patients with Bcr-Abl positive haematological malignancies, no significant myelosuppression was seen in patients with solid tumours. Non-haematological toxicities were predominantly grade 1 and included anorexia, nausea, diarrhoea, rash and fluid retention. Grade 3 hypocalcaemia was reported in one patient, perhaps reflecting inhibition of Src-dependent osteoclast function. No objective responses were reported although disease stabilisation was seen in patients with GIST, sarcoma, biliary tract cancer, mesothelioma and melanoma. Phase II evaluation of dasatinib in patients with advanced melanoma is underway [94].

\section{AZD0530}

AZD0530 (AstraZeneca, Macclesfield, UK) is a potent, selective, orally available dual inhibitor of Abl and the SFKs currently in phase I and II trials in patients with common solid tumours. Unlike dasatinib, it is not a potent inhibitor of c-Kit or the PDGFR family [63]. It also differs from dasatinib by having a longer half life enabling continuous exposure with once daily dosing in man [95]. There are cur- rently no registered trials examining the role of AZD0530 in CML or other haematological malignancies.

Clinical data demonstrate that AZD0530 inhibits the downstream effects of SFK activity (namely phosphorylation of FAK and Paxillin) in tumour tissue when delivered at tolerable doses to patients with a variety of advanced solid tumours in the phase I setting [96]. Likewise, in healthy volunteers, there is almost complete suppression of markers of bone resorption, supporting the notion that SFK inhibitors may have clinical application inhibiting normal bone turnover and malignant bone disease [97].

Pre-clinical data suggest that AZD0530 is a poor inhibitor of tumour growth, even in tumour models where potent inhibition of Src can be demonstrated [63]. However, at clinically achievable concentrations, AZD0530 inhibits a variety of in vitro models of tumour cell invasion, scattering and migration [63, 98]. In animal models of bladder and pancreatic cancer it inhibits metastasis formation with little effect on growth of the primary tumour [70, 99].

Although there are no specific data regarding AZD0530 in melanoma, its emerging good tolerability profile [95], apparent selectivity for SFKs, and its ability to inhibit its targets in a wide range of solid tumours would suggest that it may be an interesting investigational drug in this context.

\section{Bosutinib}

Bosutinib (SKI-606; Wyeth Pharmaceuticals Inc, Philadelphia, PA) is an orally available inhibitor of SFKs and Abl. Like AZD0530 it has no significant activity against PDGFR or c-Kit [100]. Preclinical data demonstrate that it can inhibit growth in models of colorectal [101-103] and breast [104] cancers. In addition, it also inhibits experimental models of invasion and cell migration in these cancers [102, 104]. It is also active in several (but not all) imatinib resistant CML cell lines [100].

Preliminary results of a phase I trial in patients with solid tumours were reported at ASCO 2007 [105]. Dose limiting toxicities were grade 3 diarrhoea and rash. Stable disease was demonstrated in patients with breast, colorectal and nonsmall cell lung cancer and one patient with pancreatic cancer achieved stable disease for greater than 52 weeks. There are currently no published data concerning the use of bosutinib in melanoma.

\section{C-KIT IN MELANOMA}

Gain of function mutations in c-Kit, a type III receptor tyrosine kinase potently inhibited by dasatinib, occur in several malignancies, most notably in the majority of GISTs, but also in some haematological malignancies and in testicular seminoma [106]. In GIST most mutations occur within exon 11 and result in in-frame deletions, insertions or point mutations in the juxtamembrane region [107]. By causing loss of function of the auto-inhibitory role of the juxtamembrane domain they facilitate ligand independent dimerisation and constitutive activation.

c-Kit is expressed by normal melanocytes and its signalling is important for melanocyte proliferation, differentiation, survival and migration [108]. However, the role, if any, of c-Kit in melanomagenesis has been controversial. Several immunohistochemical studies have reported downregulation 
of c-Kit in melanoma tissue, particularly in vertical growth phase [109, 110] and also in metastatic lesions [111]. Furthermore, expression of c-Kit in c-Kit-negative metastatic melanoma cells inhibited their growth and metastatic potential in nude mice [112]. Taken together this suggested a potential tumour or metastases suppressor role for c-Kit, perhaps via the induction of differentiation or the inhibition of growth [7]. However, recent data have added complexity to this model and suggest that, in at least a subset of melanomas, aberrant c-Kit activity may contribute to oncogenesis.

\section{Mutation or Increased Copy Number of $c$-Kit Occurs in a Subset of Melanoma}

Curtin and colleagues have recently identified $c$-Kit mutations and / or copy number increases in $39 \%$ of mucosal and $36 \%$ of acral melanomas and in $28 \%$ of melanomas arising on skin showing changes of chronic skin damage [113]. Conversely, no amplification or mutation of $c$-Kit was found in melanomas arising in skin without chronic sun damage. Sequencing of $c$-Kit in adjacent normal tissue, where possible, confirmed that mutations were somatic. BRAF and $c$-Kit mutations were essentially mutually exclusive. Interestingly, c-Kit protein levels were elevated in the majority of tumours in which $c$-Kit was mutated or amplified. A second group independently identified one of these mutations, a L576P substitution, in 3 of 20 anal melanomas [114]. Again these tumours were all strongly immuno-positive for c-Kit. A third group have also reported the L576P mutation in 2 of 100 melanomas although their subtype as per Curtin's classification was not described [115].

Identical $c$-Kit mutations are implicated in the pathogenesis of GIST, haematological malignancy and seminoma
[106]. The observation that mutant $c$-Kit appeared to be selected for further supports, albeit indirectly, a similar functional significance in melanoma. However, the effects of cKit signalling may be cell lineage-dependent. For example, expression of c-Kit with the D814Y activating mutation caused increased proliferation in mast cells whilst in melanocytes proliferation was reduced but migratory potential dramatically increased [116]. Therefore, proof-of-principle experiments to ascertain whether melanoma cell lines harbouring increased copy number or activating mutations in $c$ Kit demonstrate an enhanced sensitivity to c-Kit blockade are necessary.

\section{c-Kit Inhibition in Melanoma: Experience with Imatinib}

Imatinib is a potent, selective, small molecule inhibitor of Abl, c-Kit and PDGFR [117, 118]. In vitro, imatinib inhibited the proliferation of 8 of 19 melanoma cell lines at pharmacologically relevant concentrations [119]. Although no clear relationship between expression of c-Kit, PDGFR- $\alpha$ and PDGFR- $\beta$ existed, the two cell lines which expressed the highest levels of c-Kit were also the most sensitive to growth inhibition by imatinib. Mutations were not detected in exon 11 in any cell line although mutations elsewhere were not excluded. In vivo, imatinib was able to inhibit the growth of xenografts of B16F10 melanoma cells [120] but not of A375SM or MeWo cell lines [121]. The level of expression of c-Kit did not predict growth inhibition, nor did inhibition of PDGFR phosphorylation correlate with growth inhibition [121]. These data may indicate the involvement of alternative imatinib-sensitive kinases in melanoma growth.

Three phase II trials of imatinib in patients with advanced melanoma have been reported, all with disappointing results

Table 1. Phase 2 Studies of Imatinib in Melanoma

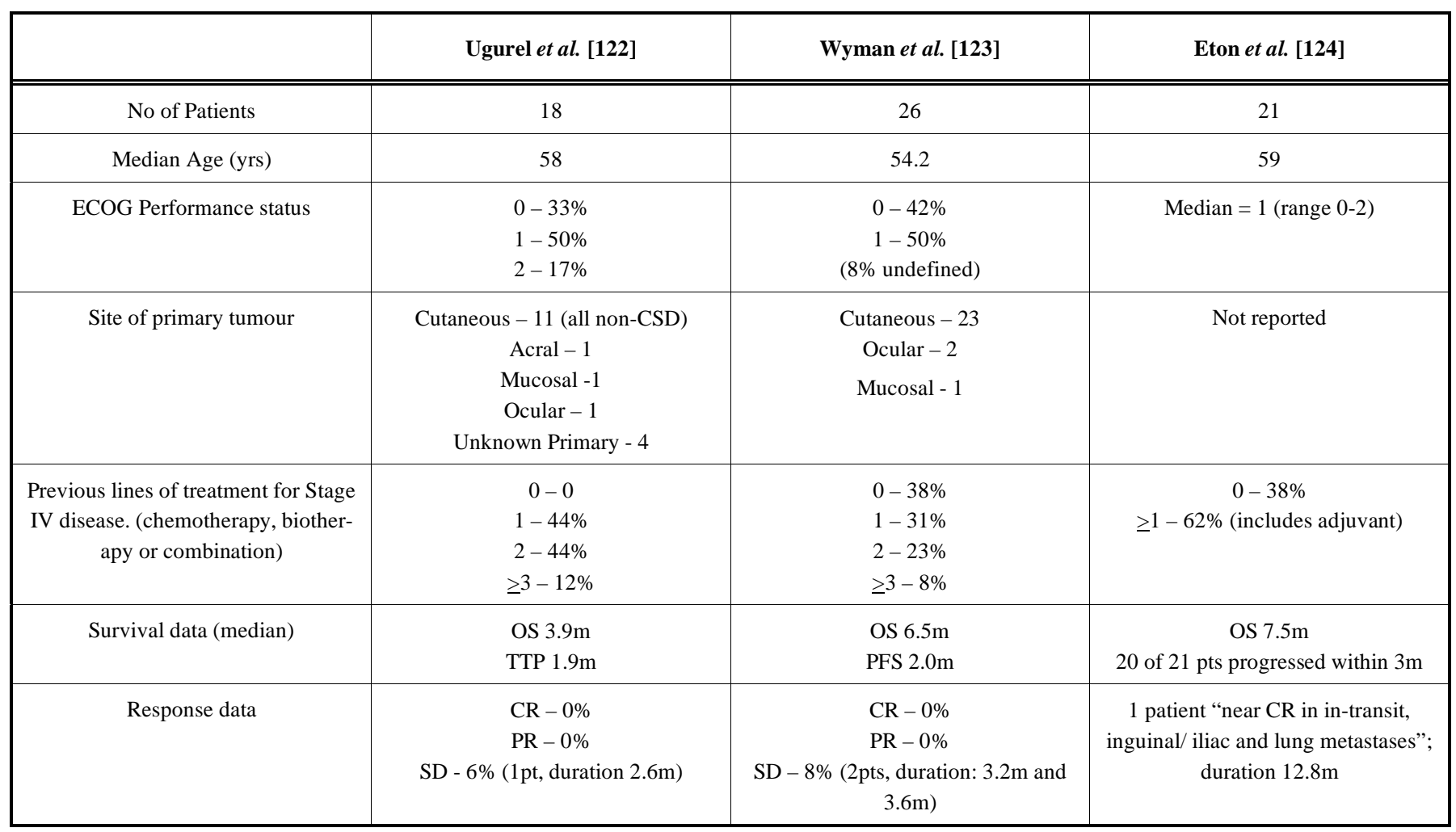

$\mathrm{OS}=$ overall survival PFS = progression free survival; $\mathrm{TTP}=$ time to progression $; \mathrm{CR}=$ complete response $\mathrm{PR}=$ partial response $; \mathrm{SD}=$ stable disease.; $\mathrm{CSD}=$ chronic sun damage. 
[122-124] (Table 1). The frequency of $c$-Kit genetic aberrations in these phase II studies has not been described. However, in Curtin's survey, increased copy number and / or mutation of $c$-Kit was usually associated with increased c-Kit protein levels [113]. In this regard the absence of strong cKit expression in all but one tumour of the 27 for which material was available in the Wyman and Ugurel studies may indicate a lack of activation of the putative target pathway. In-keeping with this observation, the patient population in at least one of these trials primarily harboured melanomas arising in skin lacking signs of chronic sun damage which would not be expected to harbour $c$-Kit aberrations [125]. Tantalisingly, the one patient in whom imatinib has induced an objective response had an acral melanoma and expressed c-Kit at a high level [124]. No $c$-Kit mutations were detected in this patient; the reported deletion of serine-715 likely represents a normal splice variant [126].

Taken together, these data support a re-evaluation of the potential role of c-Kit inhibition as a therapeutic strategy in melanoma. Should proof-of-principle preclinical data confirm the functional significance of activating mutations or increased copy number of $c$-Kit in melanoma (which seems likely by analogy to other tumours) then there is sufficient rationale to justify a further study in which patients are selected on the basis of $c$-Kit amplification or mutational status. Such a trial has already been proposed for imatinib [125]. In the existing phase II studies significant toxicity was reported, perhaps reflecting the high dose of imatinib (800 $\mathrm{mg} /$ day) in a poor prognostic group of patients. Eton et al reported a reduction in phosphorylated relative to total c-Kit as determined by dual immunofluorescence in baseline and 2nd week tumour specimens in five of five patients tested indicating that target modulation was achieved at this dose level [124]. Therefore, an important question to be addressed, with appropriate pharmacodynamic monitoring, is whether a reduced dose of imatinib may be sufficient for target inhibition but better tolerated. It should also be noted that substitutions of aspartic acid at codon 816 (comprising $12 \%$ of the c-Kit mutations reported by Curtin et al. [113]) are associated with imatinib resistance [127]. Dasatinib retains activity against D816 mutant c-Kit, albeit with some loss of potency $[79,80]$ and therefore might offer a broader spectrum of activity than imatinib in this setting. In addition, phase II studies of other multi-targeted TKIs with c-Kit activity such as sunitinib (Pfizer Inc, New York, NY) and AZD2171 (AstraZeneca, Macclesfield, UK) are already in progress in unselected patients with advanced melanoma [128].

\section{OTHER RELEVANT KINASES}

Three other tyrosine kinases known to be potently inhibited by dasatinib (although not AZD0530 or bosutinib) may be relevant to the action of this agent in melanoma and are considered briefly below:

\section{PDGFR}

Platelet-derived growth factors (PDGFs) and their receptors (PDGFRs) have been implicated in the pathogenesis of a variety of cancers and play an important role in angiogenesis [129]. Both PDGF and PDGFR are highly expressed in primary and metastatic melanoma suggesting the existence of a possible autocrine loop [130]. Additionally, the level of ex- pression of PDGFR correlated with the metastatic potential of melanoma cell lines [121]. Nevertheless, clinical experience with imatinib indicates that targeting PDGFR, at least in an unselected patient population, is unlikely to be efficacious.

\section{EPH-A2}

Although most studied for their role in development, increasing evidence links members of the ephrin family of RTKs to cancer [131]. EPH-A2, which is potently inhibited by dasatinib, has been shown to be overexpressed in many melanoma cell lines $[132,133]$ and in a subset of melanoma biopsies [134]. Ephrin-A1, a ligand for EPH-A2, was expressed in $43 \%$ of primary melanomas and $67 \%$ of metastatic melanomas [134]. In vitro, Ephrin-A1 stimulated proliferation, although not migration or invasion, of EPH-A2 expressing melanoma cell lines suggesting the potential existence of an autocrine loop in vivo [133].

\section{Abl}

Whilst the Bcr-Abl oncoprotein is responsible for the majority of cases of chronic myeloid leukaemia, a significant role for Abl signalling in solid tumours has yet to be demonstrated. Elevated levels of Abl and the related kinase Arg have been reported in breast cancer cell lines [135] and Abl signalling has been implicated in epidermal mesenchymal transition (EMT), a hallmark of invasion, in colon cancer cells [136]. Moreover, at least in some contexts Abl may be an effector of Src-induced transformation [137]. Whether and how Abl signalling may impact on melanomagenesis has not been defined.

\section{CHALLENGES IN PHASE II / III DEVELOPMENT OF SFK INHIBITORS IN MELANOMA}

Phase II trials of dasatinib in melanoma are underway or imminent in both USA and Europe. Although there are phase II programmes of AZD0530 and SKI-606 in a variety of solid tumours, there are currently no publicised studies in this indication. Whilst the commercial opportunity for new drugs in the treatment of melanoma is high, their effective and efficient development raises several challenges:

\section{Which Stage of Disease?}

As discussed above, the exact role of SFKs in melanomagenesis is not clear, but the weight of evidence in solid tumours is that their principle role is in the regulation of tumour cell invasion, rather than tumour growth. It is therefore unlikely that significant tumour shrinkage will be seen in advanced cancers. Although invasion is an ongoing process contributing to cancer morbidity and mortality at all stages of the disease, the opportunity for anti-invasive drugs, such as SFKs, may lie in the prevention of progression from early to disseminated disease. This raises the challenge of developing a drug in early disease (with the attendant cost and time implications) in the absence of a prior signal in advanced disease; whilst evidence of disease stabilisation or regression in advanced disease would present a straightforward 'go' decision in early disease, the absence of such an effect should not necessarily be seen as a 'no go' signal.

\section{Proof of Concept: Appropriate Endpoints}

Cytotoxic drug development pathways have been dependent on tumour response (shrinkage) for early decision 
making in phase II. As we do not predict tumour shrinkage as a likely outcome from SFK inhibition in melanoma, response rate is probably not an appropriate endpoint in phase II design in this setting. Indeed, even with conventional cytotoxics, objective response rates in melanoma in early phase trials do not predict subsequent regulatory approval [138]. Cytostatic endpoints such as time to progression may be valid alternatives, but these present methodological challenges. In particular, historical controls can provide misleading assumptions about anticipated tumour behaviour in untreated patients.

\section{Phase II Design Issues}

For the reasons outlined above, single-arm phase II studies of SFK inhibitors in advanced melanoma using response rate as the primary measure of efficacy are probably not appropriate. Randomized phase II designs should be considered in order that more accurate control data can be obtained, and such designs should include time to progression among the efficacy endpoints. One interesting trial design which specifically addresses disease stabilisation is the randomized discontinuation study [139], although practical implementation of this design has proved challenging in oncology [140]. Randomized designs are inevitably larger, and usually longer and more costly than single arm phase II studies.

\section{Patient Selection and Personalized Medicine}

Biomarkers which truly differentiate between patients who will and will not benefit from a given therapy are few in oncology. Nonetheless, as the number of agents increases it is essential that we continue to search for rational ways of selecting the most appropriate therapies for individual patients. This seems a tempting opportunity for molecularly targeted agents such as the SFKs, but our current understanding of the targets does not indicate a clear molecular hypothesis for rational patient selection. In particular, no data exist to support the selection of trial subjects on the basis of SFK expression or activity. Preclinical studies in breast and lung cancer have suggested an mRNA expression profile based on a small set of transcripts which is predictive of growth inhibition in cell lines [62]. However, it is not intuitive that this signature would be predictive of clinical benefit in melanoma or, fundamentally, whether such a gene signature would have relevance should clinical activity be mediated via inhibition of invasion or via effects on stromal cells.

In contrast, for c-Kit, a case for patient selection can be made; specifically to test the utility of c-Kit inhibition in the subset of patients harbouring the molecular abnormalities in c-Kit described above.

\section{Pharmacodynamic Endpoints}

Any clinical development pathway for a novel anticancer drug presents significant risk, and, as the number of patients and costs rise, the value of alternative riskminimisation strategies increases. Thus the ability to demonstrate the therapeutic principle (i.e. that the drug does inhibit the intended pathway within the tumour when administered at tolerable doses) may be important, because failure to do so could be a rational reason to terminate development in that indication. In addition, pharmacodynamic endpoints may enable rational decision making about optimal dose and schedule of a drug. The relative ease of access to cutaneous tumour deposits in melanoma should facilitate studies incorporating serial biopsies for pharmacodynamic studies. In this context potential readouts of SFK inhibition would include reduction of auto-phosphorylation of Src at tyrosine-419 as well as reduced phosphorylation of SFK substrates such as FAK and paxillin $[61,96]$.

\section{Duration of Exposure and Safety Issues}

For drugs which stabilise advanced cancer, or drugs which inhibit progression from early disease, the optimum duration of therapy may be many months or even years. All three drugs discussed are simple-to-take oral preparations but such therapy demands high levels of tolerability and safety; the exact safety and tolerability burden of these drugs in chronic administration remains largely unexplored.

\section{Combination Strategies}

A reasonably wide body of literature suggests that $\mathrm{Src}$ expression and activity is associated with acquired or innate anti-cancer drug resistance [141]. Furthermore, pharmacological inhibition of Src can overcome this resistance [67, $142,143]$. This hypothesis underpins some of the combinational drug development programmes in place for SFK inhibitors in other solid tumours, and may also be a productive approach in melanoma [23].

\section{CONCLUSIONS}

The recent discovery of SFK inhibitors and their successful progress through phase I trials offers an exciting opportunity to directly evaluate the clinical utility of SFK inhibition in melanoma. However, our understanding of the role of SFK signaling in melanoma biology remains incomplete and the successful development of SFK inhibitors in this context is by no means guaranteed. Considerable challenges lie in the design of trial strategies to reliably detect activity of an agent whose action may be primarily to inhibit invasion and metastasis rather than growth. Significantly, work to discover predictive biomarkers for SFK inhibitors is at an early stage and such information will most likely be gleaned retrospectively. Nevertheless, close cooperation between laboratory and clinic may maximize the chances of successful development of a new class of agents in this refractory disease.

\section{ACKNOWLEDGEMENTS}

We thank Val Brunton for her helpful comments.

\section{REFERENCES}

[1] Ferlay J, Bray F, Pisani P, Parkin DM. GLOBOCAN 2000: cancer incidence, mortality and prevalence worldwide, version 1.0. IARC cancerbase No.5. Lyon: IARC Press; 2001.

[2] Balch CM, Buzaid AC, Soong SJ, et al. Final version of the American Joint Committee on Cancer staging system for cutaneous melanoma. J Clin Oncol 2001; 19(16): 3635-48.

[3] Eggermont AM, Kirkwood JM. Re-evaluating the role of dacarbazine in metastatic melanoma: what have we learned in 30 years? Eur J Cancer 2004; 40(12): 1825-36.

[4] Garbe C, Eigentler TK. Diagnosis and treatment of cutaneous melanoma: state of the art 2006. Melanoma Res 2007; 17(2): 11727.

[5] Blume-Jensen P, Hunter T. Oncogenic kinase signalling. Nature 2001; 411(6835): 355-65.

[6] McArdle L, Bergin O, Fallowfield ME, Dervan PA, Easty DJ. Tyrosine phosphate in melanoma progression. Br J Dermatol 2003; 149(2): 289-95. 
[7] Easty DJ, Bennett DC. Protein tyrosine kinases in malignant melanoma. Melanoma Res 2000; 10(5): 401-11.

[8] McArdle L, Rafferty M, Maelandsmo GM, et al. Protein tyrosine phosphatase genes downregulated in melanoma. J Invest Dermatol 2001; 117(5): 1255-60.

[9] Martin GS. The road to Src. Oncogene 2004; 23(48): 7910-7.

[10] Thomas SM, Brugge JS. Cellular functions regulated by Src family kinases. Annu Rev Cell Dev Biol 1997; 13: 513-609.

[11] Roskoski R, Jr. Src protein-tyrosine kinase structure and regulation. Biochem Biophys Res Commun 2004; 324(4): 1155-64.

[12] Martin GS. The hunting of the Src. Nat Rev Mol Cell Biol 2001; 2(6): 467-75.

[13] Irby RB, Yeatman TJ. Role of Src expression and activation in human cancer. Oncogene 2000; 19(49): 5636-42.

[14] Bolen JB, Veillette A, Schwartz AM, DeSeau V, Rosen N. Activation of pp60c-src protein kinase activity in human colon carcinoma. Proc Natl Acad Sci USA 1987; 84(8): 2251-5.

[15] Cartwright CA, Meisler AI, Eckhart W. Activation of the pp60c-src protein kinase is an early event in colonic carcinogenesis. Proc Natl Acad Sci USA 1990; 87(2): 558-62.

[16] Talamonti MS, Roh MS, Curley SA, Gallick GE. Increase in activity and level of pp60c-src in progressive stages of human colorectal cancer. J Clin Invest 1993; 91(1): 53-60.

[17] Ottenhoff-Kalff AE, Rijksen G, van Beurden EA, et al. Characterization of protein tyrosine kinases from human breast cancer: involvement of the c-src oncogene product. Cancer Res 1992; 52(17): 4773-8.

[18] Verbeek BS, Vroom TM, Adriaansen-Slot SS, et al. c-Src protein expression is increased in human breast cancer. An immunohistochemical and biochemical analysis. J Pathol 1996; 180(4): 383-8.

[19] Takekura N, Yasui W, Yoshida K, et al. pp60c-src protein kinase activity in human gastric carcinomas. Int J Cancer 1990; 45(5): 847-51.

[20] Lutz MP, Esser IB, Flossmann-Kast BB, et al. Overexpression and activation of the tyrosine kinase $\mathrm{Src}$ in human pancreatic carcinoma. Biochem Biophys Res Commun 1998; 243(2): 503-8.

[21] Kumble S, Omary MB, Cartwright CA, Triadafilopoulos G. Src activation in malignant and premalignant epithelia of Barrett's esophagus. Gastroenterology 1997; 112(2): 348-56.

[22] Roskoski R, Jr. Src kinase regulation by phosphorylation and dephosphorylation. Biochem Biophys Res Commun 2005; 331(1): $1-14$.

[23] Homsi J, Messina J, Cubutt C, et al. Targeting Src/Stat3 pathway in malignant melanoma. J Clin Oncol 2006; 24(18): 459S-459S

[24] Loganzo F, Jr., Dosik JS, Zhao Y, et al. Elevated expression of protein tyrosine kinase c-Yes, but not c-Src, in human malignant melanoma. Oncogene 1993; 8(10): 2637-44.

[25] Marchetti D, Parikh N, Sudol M, Gallick GE. Stimulation of the protein tyrosine kinase c-Yes but not c-Src by neurotrophins in human brain-metastatic melanoma cells. Oncogene 1998; 16(25): 3253-60.

[26] O'Connor TJ, Neufeld E, Bechberger J, Fujita DJ. pp60c-src in human melanocytes and melanoma cells exhibits elevated specific activity and reduced tyrosine 530 phosphorylation compared to human fibroblast pp60c-src. Cell Growth Differ 1992; 3(7): 43542.

[27] Budde RJ, Ke S, Levin VA. Activity of pp60c-src in 60 different cell lines derived from human tumors. Cancer Biochem Biophys 1994; 14(3): 171-5.

[28] Niu G, Bowman T, Huang M, et al. Roles of activated Src and Stat3 signaling in melanoma tumor cell growth. Oncogene 2002; 21(46): 7001-10.

[29] Barnekow A, Paul E, Schartl M. Expression of the c-src protooncogene in human skin tumors. Cancer Res 1987; 47(1): 235-40.

[30] Wellbrock C, Lammers R, Ullrich A, Schartl M. Association between the melanoma-inducing receptor tyrosine kinase Xmrk and src family tyrosine kinases in Xiphophorus. Oncogene 1995; 10(11): 2135-43.

[31] Yayon A, Ma YS, Safran M, Klagsbrun M, Halaban R. Suppression of autocrine cell proliferation and tumorigenesis of human melanoma cells and fibroblast growth factor transformed fibroblasts by a kinase-deficient FGF receptor 1: evidence for the involvement of Src-family kinases. Oncogene 1997; 14(25): 29993009 .
[32] Irby RB, Mao W, Coppola D, et al. Activating SRC mutation in a subset of advanced human colon cancers. Nat Genet 1999; 21(2): 187-90.

[33] Daigo Y, Furukawa Y, Kawasoe T, et al. Absence of genetic alteration at codon 531 of the human c-src gene in 479 advanced colorectal cancers from Japanese and Caucasian patients. Cancer Res 1999; 59(17): 4222-4.

[34] Wang NM, Yeh KT, Tsai CH, Chen SJ, Chang JG. No evidence of correlation between mutation at codon 531 of src and the risk of colon cancer in Chinese. Cancer Lett 2000; 150(2): 201-4.

[35] Laghi L, Bianchi P, Orbetegli $\mathrm{O}$, et al. Lack of mutation at codon 531 of SRC in advanced colorectal cancers from Italian patients. Br J Cancer 2001; 84(2): 196-8.

[36] Benistant C, Chapuis H, Mottet N, et al. Deregulation of the cytoplasmic tyrosine kinase $\mathrm{cSrc}$ in the absence of a truncating mutation at codon 531 in human bladder carcinoma. Biochem Biophys Res Commun 2000; 273(2): 425-30.

[37] Sugimura M, Kobayashi K, Sagae S, et al. Mutation of the SRC gene in endometrial carcinoma. Jpn J Cancer Res 2000; 91(4): 3958.

[38] Abram CL, Courtneidge SA. Src family tyrosine kinases and growth factor signaling. Exp Cell Res 2000; 254(1): 1-13

[39] Brunton VG, Ozanne BW, Paraskeva C, Frame MC. A role for epidermal growth factor receptor, c-Src and focal adhesion kinase in an in vitro model for the progression of colon cancer. Oncogene 1997; 14(3): 283-293.

[40] Mao W, Irby R, Coppola D, et al. Activation of c-Src by receptor tyrosine kinases in human colon cancer cells with high metastatic potential. Oncogene 1997; 15(25): 3083-90.

[41] Natali PG, Nicotra MR, Di Renzo MF, et al. Expression of the cMet/HGF receptor in human melanocytic neoplasms: demonstration of the relationship to malignant melanoma tumour progression. Br J Cancer 1993; 68(4): 746-50.

[42] de Wit PE, Moretti S, Koenders PG, et al. Increasing epidermal growth factor receptor expression in human melanocytic tumor progression. J Invest Dermatol 1992; 99(2): 168-73.

[43] Sparrow LE, Heenan PJ. Differential expression of epidermal growth factor receptor in melanocytic tumours demonstrated by immunohistochemistry and mRNA in situ hybridization. Australas J Dermatol 1999; 40(1): 19-24.

[44] Otsuka T, Takayama H, Sharp R, et al. c-Met autocrine activation induces development of malignant melanoma and acquisition of the metastatic phenotype. Cancer Res 1998; 58(22): 5157-67.

[45] Meierjohann S, Schartl M. From Mendelian to molecular genetics: the Xiphophorus melanoma model. Trends Genet 2006; 22(12): 654-61.

[46] Gomez A, Wellbrock C, Gutbrod H, Dimitrijevic N, Schartl M. Ligand-independent dimerization and activation of the oncogenic Xmrk receptor by two mutations in the extracellular domain. J Biol Chem 2001; 276(5): 3333-40.

[47] Wellbrock C, Weisser C, Geissinger E, Troppmair J, Schartl M. Activation of p59(Fyn) leads to melanocyte dedifferentiation by influencing MKP-1-regulated mitogen-activated protein kinase signaling. J Biol Chem 2002; 277(8): 6443-54.

[48] Mirmohammadsadegh A, Hassan M, Bardenheuer W, et al. STAT5 phosphorylation in malignant melanoma is important for survival and is mediated through SRC and JAK1 kinases. J Invest Dermatol 2006; 126(10): 2272-80.

[49] Schaller MD, Hildebrand JD, Shannon JD, et al. Autophosphorylation of the focal adhesion kinase, pp125FAK, directs SH2dependent binding of pp60src. Mol Cell Biol 1994; 14(3): 1680-8.

[50] Thomas JW, Ellis B, Boerner RJ, et al. SH2- and SH3-mediated interactions between focal adhesion kinase and Src. J Biol Chem 1998; 273(1): 577-83

[51] Akasaka T, van Leeuwen RL, Yoshinaga IG, Mihm MC, Jr., Byers HR. Focal adhesion kinase (p125FAK) expression correlates with motility of human melanoma cell lines. J Invest Dermatol 1995; 105(1): 104-8.

[52] Hess AR, Postovit LM, Margaryan NV, et al. Focal adhesion kinase promotes the aggressive melanoma phenotype. Cancer Res 2005; 65(21): 9851-60.

[53] Masaki T, Okada M, Tokuda M, et al. Reduced C-terminal Src kinase (Csk) activities in hepatocellular carcinoma. Hepatology 1999; 29(2): 379-84

[54] Bjorge JD, Pang A, Fujita DJ. Identification of protein-tyrosine phosphatase $1 \mathrm{~B}$ as the major tyrosine phosphatase activity capable 
of dephosphorylating and activating c-Src in several human breast cancer cell lines. J Biol Chem 2000; 275(52): 41439-46.

[55] Frame MC. Src in cancer: deregulation and consequences for cell behaviour. Biochim Biophys Acta 2002; 1602(2): 114-30.

[56] Summy JM, Gallick GE. Src family kinases in tumor progression and metastasis. Cancer Metastasis Rev 2003; 22(4): 337-58.

[57] Brunton VG, MacPherson IR, Frame MC. Cell adhesion receptors, tyrosine kinases and actin modulators: a complex three-way circuitry. Biochim Biophys Acta 2004; 1692(2-3): 121-44.

[58] Huang J, Asawa T, Takato T, Sakai R. Cooperative Roles of Fyn and Cortactin in Cell Migration of Metastatic Murine Melanoma. J Biol Chem 2003; 278(48): 48367-48376.

[59] Meierjohann S, Wende E, Kraiss A, Wellbrock C, Schartl M. The oncogenic epidermal growth factor receptor variant Xiphophorus melanoma receptor kinase induces motility in melanocytes by modulation of focal adhesions. Cancer Res 2006; 66(6): 3145-52.

[60] Buettner RR, Nam S, Lee F, Jove R. Dasatinib (BMS-354825) inhibits migration and invasion of human melanoma cells and is a promising therapeutic agent for metastatic melanoma [abstract]. AACR Meeting Abstracts 2006; 2006(1): 325-d-326.

[61] Serrels A, Macpherson IR, Evans TR, et al. Identification of potential biomarkers for measuring inhibition of Src kinase activity in colon cancer cells following treatment with dasatinib. Mol Cancer Ther 2006; 5(12): 3014-22.

[62] Huang F, Reeves K, Han X, et al. Identification of candidate molecular markers predicting sensitivity in solid tumors to dasatinib: rationale for patient selection. Cancer Res 2007; 67(5): 2226-38.

[63] Green T, Hennequin LF, Ple PA, et al. Pre-clinical and early clinical activity of the highly selective, orally available, dual Src/Abl kinase inhibitor AZD0530 [abstract]. AACR Meeting Abstracts 2005; 2005(1): 1463-b-

[64] Smalley KS, Herlyn M. Targeting intracellular signaling pathways as a novel strategy in melanoma therapeutics. Ann N Y Acad Sci 2005; 1059: 16-25.

[65] Yezhelyev MV, Koehl G, Guba M, et al. Inhibition of Src Tyrosine Kinase as Treatment for Human Pancreatic Cancer Growing Orthotopically in Nude Mice. Clin Cancer Res 2004; 10(23): 8028-8036.

[66] George JA, Chen T, Taylor CC. Src Tyrosine Kinase and Multidrug Resistance Protein-1 Inhibitions Act Independently but Cooperatively to Restore Paclitaxel Sensitivity to PaclitaxelResistant Ovarian Cancer Cells. Cancer Res 2005; 65(22): 1038110388.

[67] Duxbury MS, Ito H, Zinner MJ, Ashley SW, Whang EE. Inhibition of SRC tyrosine kinase impairs inherent and acquired gemcitabine resistance in human pancreatic adenocarcinoma cells. Clin Cancer Res 2004; 10(7): 2307-18.

[68] Eliceiri BP, Paul R, Schwartzberg PL, et al. Selective requirement for Src kinases during VEGF-induced angiogenesis and vascular permeability. Mol Cell 1999; 4(6): 915-24.

[69] Kilarski WW, Jura N, Gerwins P. Inactivation of Src family kinases inhibits angiogenesis in vivo: implications for a mechanism involving organization of the actin cytoskeleton. Exp Cell Res 2003; 291(1): 70-82.

[70] Ischenko I, Guba M, Yezhelyev M, et al. Effect of Src kinase inhibition on metastasis and tumor angiogenesis in human pancreatic cancer. Angiogenesis 2007; [Epub ahead of print].

[71] Summy JM, Trevino JG, Lesslie DP, et al. AP23846, a novel and highly potent Src family kinase inhibitor, reduces vascular endothelial growth factor and interleukin-8 expression in human solid tumor cell lines and abrogates downstream angiogenic processes. Mol Cancer Ther 2005; 4(12): 1900-1911.

[72] Boyce BF, Xing L, Yao Z, et al. Src Inhibitors in Metastatic Bone Disease. Clin Cancer Res 2006; 12(20): 6291s-6295.

[73] Rucci N, Recchia I, Angelucci A, et al. Inhibition of Protein Kinase c-Src Reduces the Incidence of Breast Cancer Metastases and Increases Survival in Mice: Implications for Therapy. J Pharmacol Exp Ther 2006; 318(1): 161-172.

[74] Lombardo LJ, Lee FY, Chen P, et al. Discovery of N-(2-chloro-6methyl- phenyl)-2-(6-(4-(2-hydroxyethyl)- piperazin-1-yl)-2-methylpyrimidin-4- ylamino)thiazole-5-carboxamide (BMS-354825), a dual Src/Abl kinase inhibitor with potent antitumor activity in preclinical assays. J Med Chem 2004; 47(27): 6658-61.

[75] US National Institutes of Health, 2007. A Phase III Study of Dasatinib vs. Imatinib in Patients With Newly Diagnosed Chronic Phase CML [online]. Available from URL: http://www.clinical-
trials.gov/ct/show/NCT00481247?order=4 [Accessed 2007 Sep 24th].

[76] Shah NP, Tran C, Lee FY, et al. Overriding Imatinib Resistance with a Novel ABL Kinase Inhibitor. Science 2004; 305(5682): 399401.

[77] O'Hare T, Walters DK, Stoffregen EP, et al. In vitro activity of Bcr-Abl inhibitors AMN107 and BMS-354825 against clinically relevant imatinib-resistant Abl kinase domain mutants. Cancer Res 2005; 65(11): 4500-5.

[78] Burgess MR, Skaggs BJ, Shah NP, Lee FY, Sawyers CL. Comparative analysis of two clinically active BCR-ABL kinase inhibitors reveals the role of conformation-specific binding in resistance. Proc Natl Acad Sci USA 2005; 102(9): 3395-400.

[79] Schittenhelm MM, Shiraga S, Schroeder A, et al. Dasatinib (BMS354825), a dual SRC/ABL kinase inhibitor, inhibits the kinase activity of wild-type, juxtamembrane, and activation loop mutant KIT isoforms associated with human malignancies. Cancer Res 2006; 66(1): 473-81.

[80] Shah NP, Lee FY, Luo R, et al. Dasatinib (BMS-354825) inhibits $\mathrm{KITD} 816 \mathrm{~V}$, an imatinib-resistant activating mutation that triggers neoplastic growth in most patients with systemic mastocytosis. Blood 2006; 108(1): 286-91.

[81] Tokarski JS, Newitt JA, Chang CY, et al. The structure of Dasatinib (BMS-354825) bound to activated ABL kinase domain elucidates its inhibitory activity against imatinib-resistant ABL mutants. Cancer Res 2006; 66(11): 5790-7.

[82] Talpaz M, Shah NP, Kantarjian H, et al. Dasatinib in imatinibresistant Philadelphia chromosome-positive leukemias. N Engl J Med 2006; 354(24): 2531-41.

[83] Hochhaus A, Kantarjian HM, Baccarani M, et al. Dasatinib induces notable hematologic and cytogenetic responses in chronic-phase chronic myeloid leukemia after failure of imatinib therapy. Blood 2007; 109(6): 2303-9.

[84] Guilhot F, Apperley J, Kim DW, et al. Dasatinib induces significant hematologic and cytogenetic responses in patients with imatinib-resistant or -intolerant chronic myeloid leukemia in accelerated phase. Blood 2007; 109(10): 4143-50.

[85] Cortes J, Rousselot P, Kim DW, et al. Dasatinib induces complete hematologic and cytogenetic responses in patients with imatinibresistant or -intolerant chronic myeloid leukemia in blast crisis. Blood 2007; 109(8): 3207-13.

[86] Kantarjian H, Pasquini R, Hamerschlak N, et al. Dasatinib or highdose imatinib for chronic-phase chronic myeloid leukemia after failure of first-line imatinib: a randomized phase 2 trial. Blood 2007; 109(12): 5143-50.

[87] Shah NP. Dasatinib. Drugs Today (Barc) 2007; 43(1): 5-12.

[88] Johnson FM, Saigal B, Talpaz M, Donato NJ. Dasatinib (BMS354825) tyrosine kinase inhibitor suppresses invasion and induces cell cycle arrest and apoptosis of head and neck squamous cell carcinoma and non-small cell lung cancer cells. Clin Cancer Res 2005; 11(19 Pt 1): 6924-32.

[89] Song L, Morris M, Bagui T, et al. Dasatinib (BMS-354825) selectively induces apoptosis in lung cancer cells dependent on epidermal growth factor receptor signaling for survival. Cancer Res 2006; 66(11): 5542-8.

[90] Shor AC, Keschman EA, Lee FY, et al. Dasatinib inhibits migration and invasion in diverse human sarcoma cell lines and induces apoptosis in bone sarcoma cells dependent on SRC kinase for survival. Cancer Res 2007; 67(6): 2800-8.

[91] Nam S, Kim D, Cheng JQ, et al. Action of the Src family kinase inhibitor, dasatinib (BMS-354825), on human prostate cancer cells. Cancer Res 2005; 65(20): 9185-9.

[92] Trevino JG, Summy JM, Lesslie DP, et al. Inhibition of SRC expression and activity inhibits tumor progression and metastasis of human pancreatic adenocarcinoma cells in an orthotopic nude mouse model. Am J Pathol 2006; 168(3): 962-72.

[93] Morgan J, Demetri G, Wang D, et al. A Phase I Study of dasatinib, a SRC and multi-kinase inhibitor, in patients (pts) with GIST and other solid tumors [abstract]. Eur J Cancer 2006; 4(12): 118-118.

[94] US National Institutes of Health. Dasatinib in Treating Patients With Stage III Melanoma That Cannot Be Removed By Surgery or Stage IV Melanoma [online]. Available from URL: http:// www.clinicaltrials.gov/ct/show/NCT00436605?order=1 [Accessed 2007 Sep 24th].

[95] Gallagher NJ, Lockton AJ, Macpherson M, Marshall A, Clack G. A phase I multiple ascending dose study to assess the safety, toler- 
ability and pharmacokinetics of AZD0530, a highly selective, orally available, dual-specific Src-Abl kinase inhibitor [abstract]. AACR Meeting Abstracts 2005; 2005 (1): 935.

[96] Tabernero J, Cervantes A, Hoekman K, et al. Phase I study of AZD0530, an oral potent inhibitor of Src kinase: first demonstration of inhibition of Src activity in human cancers [abstract]. J Clin Oncol 2007; 25(18S): 3520.

[97] Boyce BF, Xing L, Yao Z, et al. SRC inhibitors in metastatic bone disease. Clin Cancer Res 2006; 12(20 Pt 2): 6291s-6295s.

[98] Hiscox S, Morgan L, Green TP, et al. Elevated Src activity promotes cellular invasion and motility in tamoxifen resistant breast cancer cells. Breast Cancer Res Treat 2006; 97(3): 263-74.

[99] Boyer B, Green T. In vivo inhibition of NBT-II bladder cell metastasis by the Src kinase inhibitor AZD0530. Clinical Cancer Research 2005; 11(24): 9024S-9024S.

[100] Puttini M, Coluccia AM, Boschelli F, et al. In vitro and in vivo activity of SKI-606, a novel Src-Abl inhibitor, against imatinibresistant Bcr-Abl+ neoplastic cells. Cancer Res 2006; 66(23): 11314-22.

[101] Boschelli DH, Wu B, Ye F, et al. Synthesis and Src kinase inhibitory activity of a series of 4-[(2,4-dichloro-5-methoxyphenyl) amino]-7-furyl-3-quinolinecarbonitriles. J Med Chem 2006; 49(26): 7868-76.

[102] Coluccia AM, Benati D, Dekhil H, et al. SKI-606 decreases growth and motility of colorectal cancer cells by preventing pp60(c-Src)dependent tyrosine phosphorylation of beta-catenin and its nuclear signaling. Cancer Res 2006; 66(4): 2279-86.

[103] Golas JM, Lucas J, Etienne C, et al. SKI-606, a Src/Abl inhibitor with in vivo activity in colon tumor xenograft models. Cancer Res 2005; 65(12): 5358-64.

[104] Jallal H, Valentino ML, Chen G, et al. A Src/Abl kinase inhibitor, SKI-606, blocks breast cancer invasion, growth, and metastasis in vitro and in vivo. Cancer Res 2007; 67(4): 1580-8.

[105] Messersmith WA, Krishnamurthi S, Hewes BA, et al. Bosutinib (SKI-606), a dual Src/Abl tyrosine kinase inhibitor: Preliminary results from a phase 1 study in patients with advanced malignant solid tumors. [abstract]. J Clin Oncol 2007; 25(18S): 3552.

[106] Heinrich MC, Blanke CD, Druker BJ, Corless CL. Inhibition of KIT Tyrosine Kinase Activity: A Novel Molecular Approach to the Treatment of KIT-Positive Malignancies. J Clin Oncol 2002; 20(6): 1692-1703.

[107] Corless CL, Fletcher JA, Heinrich MC. Biology of Gastrointestinal Stromal Tumors. J Clin Oncol 2004; 22(18): 3813-3825.

[108] Yoshida H, Kunisada T, Grimm T, et al. Review: melanocyte migration and survival controlled by $\mathrm{SCF} / \mathrm{c}$-kit expression. J Investig Dermatol Symp Proc 2001; 6(1): 1-5.

[109] Stefanou D, Batistatou A, Zioga A, et al. Immunohistochemical expression of vascular endothelial growth factor (VEGF) and CKIT in cutaneous melanocytic lesions. Int J Surg Pathol 2004; 12(2): 133-8.

[110] Montone KT, van Belle P, Elenitsas R, Elder DE. Proto-oncogene c-kit expression in malignant melanoma: protein loss with tumor progression. Mod Pathol 1997; 10(9): 939-44.

[111] Shen SS, Zhang PS, Eton O, Prieto VG. Analysis of protein tyrosine kinase expression in melanocytic lesions by tissue array. $\mathrm{J} \mathrm{Cu}-$ tan Pathol 2003; 30(9): 539-47.

[112] Luca MR, Bar-Eli M. Molecular changes in human melanoma metastasis. Histol Histopathol 1998; 13(4): 1225-31.

[113] Curtin JA, Busam K, Pinkel D, Bastian BC. Somatic activation of KIT in distinct subtypes of melanoma. J Clin Oncol 2006; 24(26): 4340-6.

[114] Antonescu CR, Busam KJ, Francone TD, et al. L576P KIT mutation in anal melanomas correlates with KIT protein expression and is sensitive to specific kinase inhibition. Int J Cancer 2007; 121(2): 257-264.

[115] Willmore-Payne C, Holden JA, Tripp S, Layfield LJ. Human malignant melanoma: detection of BRAF- and c-kit-activating mutations by high-resolution amplicon melting analysis. Hum Pathol 2005; 36(5): 486-93.

[116] Alexeev V, Yoon K. Distinctive role of the cKit receptor tyrosine kinase signaling in mammalian melanocytes. J Invest Dermatol 2006; 126(5): 1102-10.

[117] Buchdunger E, Zimmermann J, Mett H, et al. Inhibition of the Abl protein-tyrosine kinase in vitro and in vivo by a 2phenylaminopyrimidine derivative. Cancer Res 1996; 56(1): 100-4.
[118] Buchdunger E, Cioffi CL, Law N, et al. Abl protein-tyrosine kinase inhibitor STI571 inhibits in vitro signal transduction mediated by ckit and platelet-derived growth factor receptors. J Pharmacol Exp Ther 2000; 295(1): 139-45.

[119] Mayorga ME, Sanchis D, Perez de Santos AM, et al. Antiproliferative effect of STI571 on cultured human cutaneous melanomaderived cell lines. Melanoma Res 2006; 16(2): 127-35.

[120] Redondo P, Lloret P, Andreu EJ, Inoges S. Imatinib mesylate in cutaneous melanoma. J Invest Dermatol 2004; 123(6): 1208-9.

[121] McGary EC, Onn A, Mills L, et al. Imatinib mesylate inhibits platelet-derived growth factor receptor phosphorylation of melanoma cells but does not affect tumorigenicity in vivo. J Invest Dermatol 2004; 122(2): 400-5.

[122] Ugurel S, Hildenbrand R, Zimpfer A, et al. Lack of clinical efficacy of imatinib in metastatic melanoma. Br J Cancer 2005; 92(8): 1398-405.

[123] Wyman K, Atkins MB, Prieto V, et al. Multicenter Phase II trial of high-dose imatinib mesylate in metastatic melanoma: significant toxicity with no clinical efficacy. Cancer 2006; 106(9): 2005-11.

[124] Eton O, Billings L, Kim K, et al. Phase II trial of imatinib mesylate (STI-571) in metastatic melanoma (MM) [abstract]. J Clin Oncol 2004; 22(14S): 7528 .

[125] Becker JC, Brocker EB, Schadendorf D, Ugurel S. Imatinib in Melanoma: A Selective Treatment Option Based on KIT Mutation Status? J Clin Oncol 2007; 25(7): 9e.

[126] Crosier P, Ricciardi S, Hall L, et al. Expression of isoforms of the human receptor tyrosine kinase c-kit in leukemic cell lines and acute myeloid leukemia. Blood 1993; 82(4): 1151-1158.

[127] Ma Y, Zeng S, Metcalfe DD, et al. The c-KIT mutation causing human mastocytosis is resistant to STI571 and other KIT kinase inhibitors; kinases with enzymatic site mutations show different inhibitor sensitivity profiles than wild-type kinases and those with regulatory-type mutations. Blood 2002; 99(5): 1741-4.

[128] Fecher LA, Cummings SD, Keefe MJ, Alani RM. Toward a molecular classification of melanoma. J Clin Oncol 2007; 25(12): 1606-20.

[129] Ostman A. PDGF receptors-mediators of autocrine tumor growth and regulators of tumor vasculature and stroma. Cytokine Growth Factor Rev 2004; 15(4): 275-86.

[130] Barnhill RL, Xiao M, Graves D, Antoniades HN. Expression of platelet-derived growth factor (PDGF)-A, PDGF-B and the PDGFalpha receptor, but not the PDGF-beta receptor, in human malignant melanoma in vivo. Br J Dermatol 1996; 135(6): 898-904.

[131] Dodelet VC, Pasquale EB. Eph receptors and ephrin ligands: embryogenesis to tumorigenesis. Oncogene 2000; 19(49): 5614-9.

[132] Easty DJ, Herlyn M, Bennett DC. Abnormal protein tyrosine kinase gene expression during melanoma progression and metastasis. Int J Cancer 1995; 60(1): 129-36.

[133] Easty DJ, Guthrie BA, Maung K, et al. Protein B61 as a new growth factor: expression of B61 and up-regulation of its receptor epithelial cell kinase during melanoma progression. Cancer Res 1995; 55(12): 2528-32.

[134] Easty DJ, Hill SP, Hsu MY, et al. Up-regulation of ephrin-A1 during melanoma progression. Int J Cancer 1999; 84(5): 494-501.

[135] Srinivasan D, Plattner R. Activation of Abl tyrosine kinases promotes invasion of aggressive breast cancer cells. Cancer Res 2006 66(11): 5648-55

[136] Yang L, Lin C, Liu ZR. P68 RNA helicase mediates PDGFinduced epithelial mesenchymal transition by displacing Axin from beta-catenin. Cell 2006; 127(1): 139-55

[137] Sirvent A, Boureux A, Simon V, Leroy C, Roche S. The tyrosine kinase $\mathrm{Abl}$ is required for Src-transforming activity in mouse fibroblasts and human breast cancer cells. Oncogene 2007; [Epub ahead of print].

[138] Goffin J, Baral S, Tu D, Nomikos D, Seymour L. Objective Responses in Patients with Malignant Melanoma or Renal Cell Cancer in Early Clinical Studies Do Not Predict Regulatory Approval. Clin Cancer Res 2005; 11(16): 5928-5934.

[139] Rosner GL, Stadler W, Ratain MJ. Randomized discontinuation design: application to cytostatic antineoplastic agents. J Clin Oncol 2002; 20(22): 4478-84.

[140] Sonpavde G, Hutson TE, Galsky MD, Berry WR. Problems with the randomized discontinuation design. J Clin Oncol 2006; 24(28): 4669-70; author reply 4670-1. 
[141] Shah AN, Gallick GE. Src, chemoresistance and epithelial to mesenchymal transition: are they related? Anticancer Drugs 2007; 18(4): 371-5.

[142] Phillips K, Parikh N, Kuwai T, Green T, Gallick GE. Inhibition of colon tumor metastasis in an orthotopic nude mouse model with the dual selective Src/Abl kinase inhibitor, ADZ0530. [abstract]. J Clin Oncol 2007; 25(18S): 14032.

[143] Hiscox S, Green TP, Smith C, et al. Effectiveness of the dual specific Src/Abl kinase inhibitor AZD0530 in combination with tamoxifen in preventing acquired anti-estrogen resistance in breast cancer cells. [abstract]. J Clin Oncol 2007; 25(18S): 14054. 